Quintas-Froufe, Natalia (2016). Indicadores de calidad de las publicaciones cientificas en el área de Ciencias Sociāes en España: un análisis comparativo entre agencias evaluadoras. Revista de Investigación Educativa, 34(1), 259-272. DOI: http://dx.doi.org/10.6018/rie.34.1.210191

\title{
Indicadores de calidad de las publicaciones científicas en el área de Ciencias Sociales en España: un análisis comparativo entre agencias evaluadoras
}

\author{
Quality Indicators for Scientific Publications in the Social Sciences \\ in Spain: a Comparative Analysis between Evaluation Agencies
}

\author{
Natalia Quintas-Froufe \\ Universidade da Coruña
}

\begin{abstract}
Resumen
Las publicaciones científicas (artículos y libros) son una de las dimensiones básicas de la evaluación de la calidad de la investigación del profesorado universitario en España. En este artículo se describen, analizan y comparan los indicadores de calidad que los tres organismos principales de evaluación de la actividad científica del profesorado universitario español emplean a la hora de evaluar las publicaciones científicas en el área de Ciencias Sociales. Las entidades seleccionadas son: la Agencia Nacional de Evaluación de la Calidad y Acreditación (ANECA), la Agencia Nacional de Evaluación y Prospectiva (ANEP) y la Comisión Nacional Evaluadora de la Actividad Investigadora (CNEAI). Los resultados muestran que el criterio común de calidad más destacable para los artículos es su publicación en revistas incluidas en el Journal Citation Reports (JCR) o en SCOPUS. Se concluye, por tanto, que las bases de datos bibliográficas y bibliométricas internacionales son los indicadores de calidad unánimemente aceptados.

Palabras clave: publicación científica, indicadores de calidad, ciencias sociales, bases bibliográficas.
\end{abstract}

Correspondencia: Natalia Quintas-Froufe. Universidade da Coruña. Facultad de Ciencias de la Comunicación 15007 Campus de Elviña (A Coruña) n.quintas.froufe@udc.es 


\begin{abstract}
Scientific publications (articles and books) are one of the basics for evaluating research. This article describes, analyzes and compares the quality criteria employed by the three major agencies which evaluate the scientific activity of Spanish university faculty when evaluating publications in the area of Social Sciences. The three selected entities are: ANECA (National Agency for Quality Assessment and Accreditation of Spain), ANEP (National Evaluation and Planning Agency) and CNEAI (National Research Activity Evaluation Commission). The results show that the most important quality criterion is the publication of articles in journals included in the Journal Citation Reports and SCOPUS. The conclusion reached was that international bibliographic and bibliometric databases are the only unanimously accepted quality indicators.

Keywords: scientific publication, quality indicators, social sciences, bibliographic databases.
\end{abstract}

\title{
Introducción
}

La evaluación de la actividad científica del profesorado universitario español se rige por los criterios establecidos por los principales organismos de evaluación: la Agencia Nacional de Evaluación de la Calidad y Acreditación (ANECA), encargada de la acreditación del profesorado universitario; la Agencia Nacional de Evaluación y Prospectiva (ANEP) evaluadora de los grupos de investigación y la calidad de los proyectos; y la Comisión Nacional Evaluadora de la Actividad Investigadora (CNEAI), responsable de la evaluación de la actividad investigadora y de otorgar los tramos de investigación.

El planteamiento de esta investigación parte de que, como sostienen Torres-Salinas, Bordons, Giménez-Toledo, Delgado-López-Cózar, Jiménez-Contreras y Sanz-Casado (2010) "los criterios de las agencias son por tanto un punto de partida necesario a la hora de detectar cuáles son las herramientas de evaluación que más consenso generan entre la comunidad científica y aquellas que sin duda deberían considerarse en las construcción de indicadores bibliométricos al ser esenciales en la política científica española" (p. 677).

En el marco de dicha actividad evaluadora, las publicaciones científicas (entendidas como los artículos publicados en revistas indexadas y no indexadas y los libros procedentes de trabajos de investigación previos) adquieren un peso significativo en el currículum del profesor universitario e incluso "la evaluación de los artículos y las revistas se ha convertido en la columna vertebral de todo el proceso de evaluación de la calidad investigadora de una nación" (Hernández y Maquilón, 2010, p. 15). Además, la calidad de estas determinará poderosamente la promoción en la carrera académica recayendo en ellas la base principal de la evaluación, tal como han confirmado Galán, González-Galán y Rodríguez-Patrón (2014) en su reciente investigación sobre la evaluación de la actividad del profesorado universitario en España: "[...] el 65-70\% del peso recae en las publicaciones, con un claro acento en los artículos indexados con factor de impacto (especialmente en el Journal Citation Reports [JCR] de la Web of Science de Thomson Reuters)" (p. 144). 
La relevancia que estas tres instituciones conceden a estas publicaciones (concretamente a los indicadores de impacto de los artículos publicados en revistas indexadas) en el proceso de evaluación del profesorado hace que el análisis de los criterios de calidad de las mismas sea un tema de máximo interés en la actualidad y haya suscitado gran debate entre los investigadores. Por una parte, en investigaciones anteriores se han analizado los principios y criterios utilizados en España por la Comisión Nacional Evaluadora de la Actividad Investigadora (CNEAI) para la valoración de las publicaciones científicas durante el periodo 1989-2009 (Ruíz-Pérez, Delgado-López-Cózar y Jiménez-Contreras, 2010); la evaluación de los tramos de investigación en el área de Economía y Empresa (Cancelo y Bastida, 2013); la labor de evaluación de la Agencia Nacional de Evaluación y Prospectiva (Caride, 2011); o la valoración como indicio de calidad en la normativa evaluadora de las revistas españolas de Ciencias Sociales en el Journal Citation Reports (Moreno-Pulido, López-González, Rubio-Garay, Ángel Saúl y Sánchez-Elvira-Paniagua, 2013).

Por otra parte, se ha cuestionado la influencia de las políticas de evaluación de estos organismos sobre la actividad investigadora del profesorado. Por ejemplo, JiménezContreras, De Moya Anegón y Delgado-López-Cózar (2003), analizaron el impacto del sistema de evaluación de la CNEAI en la producción científica española; Galán y Zych (2011) llevaron a cabo ese mismo análisis en el campo de la Educación; Soriano (2008) acuñó el término "efecto Aneca" al detectar el influjo de dicha agencia sobre los contenidos de la actividad investigadora de los potenciales evaluados; Masip (2011) analizó el "efecto Aneca" en la producción española en comunicación en el Social Science Sitation Index; y Perceval y Fornieles (2008) estudiaron "la perversa unión entre la publicación de los conocimientos en ciencias sociales y humanidades, y la valoración de los mismos por tribunales académicos con vistas a la acreditación para acceder a los puestos y cargos universitarios" (p. 213).

Sin embargo, a día de cierre de esta investigación, todavía no se ha publicado un trabajo que exponga los criterios de calidad de los tres organismos de forma actualizada y delimitada a un área específica de conocimiento, las Ciencias Sociales. El interés en el área de Ciencias Sociales está determinado por las particularidades de la misma: un área sin tradición y vocación internacional, donde la incorporación de esos criterios internacionales se está realizando de forma paulatina. Un área de conocimiento en la que se aplican los criterios de otras áreas, principalmente de las ciencias, con mayor tradición investigadora. Diferentes autores han hecho hincapié en su particular idiosincrasia y también en las características de los investigadores en Ciencias Sociales y Humanidades (Castro, Barrenechea e Ibarra, 2006; Delgado-López-Cózar, OrduñaMalea, Jiménez-Contreras y Ruiz-Pérez, 2014; Delgado-López-Cózar y Ruiz-Pérez, 2009; Rovira, 2006), por ejemplo:

publicar en una amplia variedad de medios, aunque con una especial predilección por las monografías: libros y capítulos de libros; utilizar su lengua vernácula como principal medio de expresión y publicar fundamentalmente en medios nacionales, bien sean revistas, actas de congresos o editoriales de monografías (Delgado-López-Cózar et al., 2014, pp. 88-89). 


\section{Objetivo del estudio y metodología}

El objetivo de este artículo es identificar, analizar y comparar los principales indicadores de calidad que utilizan los tres organismos (ANECA, ANEP y CNEAI) de evaluación de la actividad científica del profesorado española la hora de valorar las publicaciones científicas en el área de Ciencias Sociales con el fin de establecer un análisis descriptivo-comparativo entre ambos para identificar la posible existencia o no de un cierto consenso a la hora de utilizar los mismos indicadores ( $y$, secundariamente, las mismas fuentes).

\section{Procedimiento}

El método de investigación seleccionado es de carácter inductivo-deductivo y sigue un proceso analítico-sintético con el fin de identificar, comparar y sintetizar los hallazgos de la investigación. La recopilación de los datos se realizó durante el mes de diciembre de 2014 a partir de las fuentes documentales legislativas de las tres instituciones. En primer lugar se muestra la descripción de los criterios utilizados para finalmente aportar el análisis comparativo plasmado en tres matrices de datos de elaboración propia.

Cabe destacar que en el año 2012, la ANECA publicó un documento "Principios y Orientaciones 2.0" con las bases de la nueva política de evaluación del profesorado. Posteriormente se decidió volver a la anterior versión, que es la única que se tiene en cuenta en esta investigación al ser la vigente.

\section{La Agencia Nacional de Evaluación de la Calidad y Acreditación (ANECA): principales indicadores}

La ANECA, junto con las agencias autonómicas de evaluación, es el organismo responsable de la acreditación del profesorado universitario. Sin embargo, las evaluaciones de este organismo son las únicas válidas para todo el territorio español, de ahí que se descartara el análisis de las políticas de evaluación de las agencias de evaluación autonómicas.

La ANECA tiene en marcha varios programas para evaluar los perfiles de los solicitantes en función de su actividad docente e investigadora: el programa ACADEMIA: programa de acreditación nacional para el acceso a los cuerpos docentes universitarios (profesores titulares de Universidad y catedráticos de Universidad); y el programa PEP: programa de evaluación del profesorado para la contratación de profesor contratado (profesor ayudante doctor, profesor contratado doctor, y profesor de Universidad privada). Para esta investigación se toman como referencia ambos programas, en los cuales se mencionan referencias de calidad propias del área de Ciencias Sociales con baremos que la diferencian de las demás áreas debido a la singularidad de la misma. Cabe destacar el análisis que aportan las autoras Ortiz-de-Urbina-Criado y MoraValentín (2013) sobre la evolución y los cambios del programa ACADEMIA desde su implantación hasta el año 2013.

En primer lugar es preciso identificar el tipo de publicaciones científicas que se valoran: artículos (en revistas indexadas y no indexadas) y libros y capítulos de libros (resultados de un proceso de investigación). En el programa ACADEMIA se distingue 
entre revistas indexadas (en catálogos que asignen a cada revista un índice de calidad relativo dentro del conjunto de revistas asociadas a una misma categoría: Journal Citation Reports o equivalentes en cada especialidad) y no indexadas (ANECA, 2008). Se valora que las revistas indexadas estén en posiciones relevantes en los listados por ámbitos científicos en el Subject Category Listing del Journal Citation Reports del Science Citation Index (SCI), del Social Sciences Citation Index (SSCI) y del Arts and Humanities Citation Index (AHCI), en el Philosopher's Index, en el Répertoire Bibliographique de Louvain o similares. Como referencias propias del área de Ciencias Sociales y Jurídicas se incluyen otros índices como Econlit, catálogo de Latindex, o la base de datos DICE (Difusión y Calidad Editorial de las Revistas Españolas de Humanidades y Ciencias Sociales y Jurídicas).

Además, se añaden otros indicadores de calidad como: el índice de impacto, el lugar que ocupa la revista en el conjunto de las que corresponden a un mismo ámbito de conocimiento, el número de autores y, cuando sea relevante, la posición que ocupa entre ellos el solicitante. En el programa PEP, también se incorporan los siguientes criterios para valorar este tipo de publicaciones científicas: el tiempo transcurrido desde la lectura de la tesis doctoral y la coherencia de una línea de investigación bien definida y mantenida a lo largo del tiempo (los cambios a nuevas líneas, con resultados satisfactorios, no se consideran de forma negativa).

Los criterios de calidad para las publicaciones científicas no indexadas, adoptados en ambos programas, son: a) la calidad informativa (identificación de los comités editoriales y científicos, instrucciones a autores, información sobre el proceso de evaluación y selección de manuscritos, traducción de sumarios, títulos de los artículos, palabras claves, resúmenes en inglés y publicación de datos del proceso editorial); b) la calidad del proceso editorial (periodicidad, regularidad, arbitraje científico, revisores, anonimato en la revisión, instrucciones para la revisión, comunicación motivada de las decisiones, consejos de redacción y asesor); c) la calidad científica (porcentaje y tasa de aceptación de artículos de investigación) y d) la calidad de la difusión y visibilidad (inclusión en bases bibliográficas).

En relación a otro tipo de publicaciones que se tienen en cuenta en ambos programas, como son los libros y capítulos de libros, únicamente se valoran los que posean ISBN (en el programa PEP este no es un requisito ineludible sino un valor añadido) y estén publicados en editoriales especializadas de reconocido prestigio donde se garantice un riguroso proceso de selección y evaluación de trabajos. Los criterios de calidad son: el número y calidad de las citas (u otras medidas de nivel de impacto), el prestigio internacional de la editorial, los editores, la colección en la que se publica, el rigor en el proceso de selección y evaluación de originales y las reseñas en las revistas científicas especializadas. Cabe mencionar que en el programa PEP el criterio de calidad únicamente es el número de citas (no la calidad de las mismas) aunque se añaden también otras cuestiones de valoración como la extensión y las traducciones a otras lenguas.

\section{La Agencia Nacional de Evaluación y Prospectiva (ANEP): principales indicadores}

La ANEP es una unidad dependiente de la Dirección General de Investigación Científica y Técnica y la responsable de evaluar a los grupos de investigación para su 
financiación y la calidad de los proyectos, así como el currículum del solicitante. Los criterios de evaluación que emplea son publicados en la propia página web, en las convocatorias específicas (por ejemplo las ayudas para contratos Juan de la Ciervaformación y Ayudas para contratos Ramón y Cajal) o en órdenes ministeriales. El área de Ciencias Sociales aborda objetivos científicos relacionados con las disciplinas de Análisis Geográfico Regional, Antropología Social, Biblioteconomía y Documentación, Ciencia Política y de la Administración, Comunicación Audiovisual y Publicidad, Geografía Humana, Historia del Pensamiento y de los Movimientos Sociales, Periodismo, Sociología, Trabajo Social y Servicios Sociales, así como Urbanismo y Ordenación del Territorio (ANEP, 2007).

En 2007 la ANEP constituyó un grupo de trabajo en Ciencias Sociales para establecer guías básicas de evaluación para los investigadores en esta área. En relación a la tipología de las publicaciones se diferencia entre periódicas y no periódicas. Para las primeras se tiene en cuenta "el ISI" o, "en su defecto, se tomarán también en consideración artículos publicados en revistas que cumplan criterios de calidad similares a los de las revistas de los listados elaborados por el ISI" (ANEP, 2007, p. 7). Como otros indicadores de calidad se incluye el índice de impacto de la revista y las citas que el artículo haya recibido, que serán valorados en función de la productividad media mundial del área y de la especialidad. Se señala explícitamente la preocupación por utilizar bases de datos nacionales (se menciona al desaparecido INRECS: Índice de impacto de las Revistas Españolas de Ciencias Sociales elaborado por el Grupo de Investigación EC3 sobre Evaluación de la Ciencia y de la Comunicación científica de la Universidad de Granada) en la evaluación de la investigación ya que al ceñirse al ámbito nacional puede dar lugar a malinterpretaciones. Se menciona, por ejemplo, el hecho de que "al considerar únicamente el ámbito nacional, las revistas españolas incluidas en el ISI no resultan, en general, bien clasificadas en estos índices bibliométricos nacionales, pues sus artículos deberían considerarse en el ámbito internacional, mucho más competitivo y abierto" (ANEP, 2007, p. 14). En consecuencia, se recomienda impulsar la incorporación de las revistas españolas a bases internacionales en vez de crear listados nacionales.

En relación a las publicaciones no periódicas, se refieren únicamente a las monografías y capítulos de libros. El principal criterio de valoración es la calidad de la editorial. Para juzgarla se tienen en cuenta los procesos de evaluación y selección de los originales, y las indicaciones sobre su impacto (número de citas en publicaciones relevantes; artículos en revistas ISI o similares, otras monografías de calidad y análogos).

Aunque las citas son un criterio común de calidad de las publicaciones periódicas y no periódicas, se considera que pueden ser indicadores "defectuosos" en el corto plazo y en función de las áreas. Por ello abogan por recurrir a un panel de expertos para su evaluación.

Los expertos son investigadores de calidad y activos, esto es, deben tener reconocido prestigio en su área, con proyección internacional y nacional, que debe estar acreditada por el impacto de sus publicaciones, y por poseer una cierta trayectoria, suficiente como para poder aplicar índices algo más sofisticados, como por ejemplo, el índice $\mathrm{h}$-por encima de la mediana de su área-, proyectos de investigación, y, si procede, poseer sexenios obtenidos en convocatorias recientes (los últimos 7 años) (ANEP, 2007, p. 15). 


\section{La Comisión Nacional Evaluadora de la Actividad Investigadora (CNEAI): principales indicadores}

La CNEAI es el organismo responsable de valorar anualmente la actividad investigadora del profesorado universitario otorgando los tramos de investigación. En esta investigación se hace referencia a la última resolución hasta la fecha (de 26 de noviembre de 2014 publicada en el «BOE» núm. 290, de 1 de diciembre de 2014) para el campo número 7 (Ciencias Sociales, Políticas, del Comportamiento y de la Educación) y 8 (Ciencias Económicas y Empresariales). Las publicaciones científicas que se valoran son los artículos (impresos y electrónicos), libros y capítulos de libros (como resultado de un proceso de investigación).

En relación a los artículos, el primer indicador de calidad es que hayan sido publicados en revistas indexadas en el Journal Citation Reports (Social Science Citation Index yScience Citation Index) del Web of Science. En segundo lugar, también se valoran las publicaciones incluidas en los listados de SCOPUS y en Arts and Humanities Citation Index del Web of Science, en revistas indexadas en otras bases de datos nacionales o internacionales (por ejemplo, ERIH, CIRC, DICE-CINDOC, etc.), o en aquellas revistas acreditadas por la FECYT (Fundación Española para Ciencia y Tecnología). Estas últimas, se puntualiza, tendrán una calidad similar a las indexadas en las bases anteriores y además tendrán que satisfacer los siguientes requisitos en relación a: a) la calidad informativa de la revista como medio de comunicación científica, b) la calidad del proceso editorial y c) la calidad científica de las revistas.

Cabe destacar que en las áreas de Sociología, de Ciencia Política y de la Administración, de Ciencias de la Educación y de Comunicación se valora por igual los artículos publicados en el Journal Citation Reports o en SCOPUS y en el Arts and Humanities Citation Index del Web of Science, exigiéndose dos publicaciones como mínimo (de las cinco exigidas) con estos criterios para poder optar a una valoración positiva. Sin embargo, en las áreas de Psicología y Biblioteconomía y Documentación se elimina esta equivalencia y se requiere que cuatro de las aportaciones sean artículos publicados en el Journal Citation Reports, y tres de ellos en los cuartiles primero y segundo de su categoría. En estas áreas también se especifica que se podrá obtener la evaluación positiva atendiendo a la cantidad y relevancia de las citas recibidas por las aportaciones cuando sean especialmente destacables.

Sin embargo, en el campo 8 (Ciencias Económicas y Empresariales) es necesario que las cinco aportaciones ocupen posiciones relevantes en el Journal Citation Reports (JCR) o SCOPUS y dos de las contribuciones deben estar publicadas en revistas indexadas en JCR (Social Sciences Edition o Science Edition) con relevancia significativa para este campo.

Respecto a los libros y capítulos, los criterios de calidad son: "el número de citas recibidas; el prestigio de la editorial; los editores; la colección en la que se publica la obra; las reseñas en las revistas científicas especializadas, y las traducciones de la propia obra a otras lenguas" (Boletín Oficial del Estado, 2014).

\section{Resultados}

Los tres organismos coinciden en señalar al antiguo ISI (actualmente Thomson Reuters) como criterio de calidad de las revistas científicas. Concretamente, la ANECA considera al Journal Citation Reports del Science Citation Index y Social Sciences Citation 
Index como el principal indicador de calidad de las revistas científicas mientras que la CNEAI también parte de este planteamiento aunque equipara en algunas áreas, como por ejemplo Sociología, Ciencia Política y de la Administración, Ciencias de la Educación o Comunicación, el Journal Citation Reports con SCOPUS.

Conviene destacar que, por una parte, las publicaciones científicas indexadas en bases de datos nacionales no son aceptadas por la ANEP (2007), mientras que la CNEAI y la ANECA reconocen las revistas recogidas en índices como el elaborado por FECYT o el desactualizado DICE-CINDOC. A continuación se exponen en la tabla 1, los índices y bases aceptadas por las tres instituciones para las publicaciones indexadas, no indexadas y los libros y capítulos de libros. Cabe destacar que la ausencia de señalización implica la no contemplación del criterio.

Tabla 1

Índices y bases bibliográficas aceptadas para las publicaciones indexadas según ANECA, ANEP y CNEAI

\begin{tabular}{|c|c|c|c|}
\hline Índices y bases & $\begin{array}{c}\text { ANECA } \\
\text { (Agencia } \\
\text { Nacional de } \\
\text { Evaluación de } \\
\text { la Calidad y } \\
\text { Acreditación) }\end{array}$ & $\begin{array}{c}\text { ANEP } \\
\text { (Agencia } \\
\text { Nacional de } \\
\text { Evaluación y } \\
\text { Prospectiva) }\end{array}$ & $\begin{array}{c}\text { CNEAI } \\
\text { (Comisión } \\
\text { Nacional Eva- } \\
\text { luadora de la } \\
\text { Actividad In- } \\
\text { vestigadora) }\end{array}$ \\
\hline Science Citation Index & $X$ & $X$ & $X$ \\
\hline Social Sciences Citation Index & $X$ & $X$ & $X$ \\
\hline Arts and Humanities Citation Index & $X$ & & $X$ \\
\hline Catálogo Latindex & $X$ & & \\
\hline $\begin{array}{l}\text { CIRC (Clasificación Integrada de revistas } \\
\text { científicas) }\end{array}$ & & & $X$ \\
\hline $\begin{array}{l}\text { DICE(Difusión y Calidad Editorial de las } \\
\text { Revistas Españolas de Humanidades y } \\
\text { Ciencias Sociales y Jurídicas) }\end{array}$ & $X$ & & $X$ \\
\hline Econlit & $X$ & & \\
\hline $\begin{array}{l}\text { ERIH (European Reference Index for the } \\
\text { Humanities) }\end{array}$ & & & $X$ \\
\hline $\begin{array}{l}\text { FECYT (Fundación Española para Ciencia y } \\
\text { Tecnología) }\end{array}$ & & & $X$ \\
\hline Philosopher's Index & $X$ & & \\
\hline Répertoire Bibliographique de Louvain & $X$ & & \\
\hline SCOPUS & & & $X$ \\
\hline
\end{tabular}

Nota: Adaptado de ANECA (Agencia Nacional de Evaluación de la Calidad y Acreditación), ANEP (Agencia Nacional de Evaluación y Prospectiva) y BOE (Boletín Oficial del Estado).

Por otra parte, respecto a las publicaciones no indexadas en las bases anteriores, ANECA y CNEAI coinciden en la mayoría de los indicadores de calidad que emplean, tal como puede observarse en la tabla 2. Por el contrario, la ANEP no incluye ningún criterio de calidad para este tipo de publicaciones. 
Tabla 2

Indicadores de calidad para las publicaciones no indexadas según ANECA y CNEAI

\begin{tabular}{|c|c|c|c|}
\hline & Indicadores & $\begin{array}{c}\text { ANECA } \\
\text { (Agencia Nacional de } \\
\text { Evaluación de la Cali- } \\
\text { dad y Acreditación) }\end{array}$ & $\begin{array}{c}\text { CNEAI } \\
\text { (Comisión Nacional } \\
\text { Evaluadora de la Acti- } \\
\text { vidad Investigadora) }\end{array}$ \\
\hline \multirow{5}{*}{$\begin{array}{c}\text { Calidad } \\
\text { informativa }\end{array}$} & $\begin{array}{l}\text { Identificación de los comités edi- } \\
\text { toriales y científicos }\end{array}$ & $X$ & $X$ \\
\hline & Instrucciones a autores & $X$ & $X$ \\
\hline & $\begin{array}{l}\text { Información sobre el proceso de } \\
\text { evaluación y selección de manus- } \\
\text { critos }\end{array}$ & $X$ & $X$ \\
\hline & $\begin{array}{l}\text { Traducción de sumarios, títulos } \\
\text { de los artículos, palabras claves, } \\
\text { resúmenes en inglés }\end{array}$ & $X$ & $X$ \\
\hline & $\begin{array}{l}\text { Publicación de datos del proceso } \\
\text { editorial }\end{array}$ & $X$ & $X$ \\
\hline \multirow{9}{*}{$\begin{array}{l}\text { Calidad } \\
\text { del proceso } \\
\text { editorial }\end{array}$} & Periodicidad & $X$ & $X$ \\
\hline & Regularidad & $X$ & $X$ \\
\hline & Arbitraje científico & $X$ & $X$ \\
\hline & $\begin{array}{l}\text { Homogeneidad de la línea edito- } \\
\text { rial (en editoriales de libros) }\end{array}$ & & $X$ \\
\hline & Revisores & $X$ & $X$ \\
\hline & Anonimato en la revisión & $X$ & $X$ \\
\hline & Instrucciones para la revisión & $X$ & $X$ \\
\hline & $\begin{array}{l}\text { Comunicación motivada de las } \\
\text { decisiones }\end{array}$ & $X$ & $X$ \\
\hline & Consejos de redacción y asesor & $X$ & $X$ \\
\hline \multirow[b]{2}{*}{$\begin{array}{l}\text { Calidad } \\
\text { Científica }\end{array}$} & $\begin{array}{l}\text { Porcentaje y tasa de aceptación } \\
\text { de artículos de investigación }\end{array}$ & $X$ & $\begin{array}{l}>75 \% \text { de los artículos } \\
\text { deberán ser trabajos que } \\
\text { comuniquen resultados de } \\
\text { investigación originales }\end{array}$ \\
\hline & $\begin{array}{l}\text { Autoría: grado de endogamia edi- } \\
\text { torial, más del } 75 \% \text { de los autores } \\
\text { serán externos al comité editorial } \\
\text { y virtualmente ajenos a la orga- } \\
\text { nización editorial de la revista. }\end{array}$ & & 0 \\
\hline $\begin{array}{l}\text { Calidad de } \\
\text { la difusión y } \\
\text { visibilidad }\end{array}$ & Inclusión en bases bibliográficas & $X$ & \\
\hline
\end{tabular}


Y por último, en relación a los libros y capítulos de libros, las citas y el prestigio de la editorial son los dos indicadores que los tres organismos coinciden en señalar (Tabla 3).

Tabla 3

Indicadores de calidad para los libros y capítulos de libros

\begin{tabular}{lccc}
\hline \multicolumn{1}{c}{ Indicadores } & $\begin{array}{c}\text { ANECA } \\
\text { (Agencia } \\
\text { Nacional de } \\
\text { Evaluación de } \\
\text { la Calidad y } \\
\text { Acreditación) }\end{array}$ & $\begin{array}{c}\text { ANEP } \\
\text { (Agencia } \\
\text { Nacional de } \\
\text { Evaluación y } \\
\text { Prospectiva) }\end{array}$ & $\begin{array}{c}\text { CNEAI } \\
\text { (Comisión } \\
\text { Nacional } \\
\text { Evaluadora de } \\
\text { la Actividad } \\
\text { Investigadora) }\end{array}$ \\
\hline $\begin{array}{l}\text { Número y calidad de las citas } \\
\begin{array}{l}\text { Prestigio internacional de la editorial } \\
\text { Editores }\end{array}\end{array}$ & $X$ & $X$ & $X$ \\
$\begin{array}{l}\text { Colección en la que se publica } \\
\text { Rigor en el proceso de selección } y\end{array}$ & $X$ & $X$ & $X$ \\
evaluación de originales & $X$ & & $X$ \\
$\begin{array}{l}\text { Reseñas en las revistas cientificas } \\
\text { especializadas }\end{array}$ & $X$ & $X$ & $X$ \\
$\begin{array}{l}\text { Traducciones de la propia obra a } \\
\text { otras lenguas }\end{array}$ & & $X$ & $X$ \\
\hline
\end{tabular}

Nota: Adaptado de ANECA (Agencia Nacional de Evaluación de la Calidad y Acreditación), CNEAI (Comisión Nacional Evaluadora de la Actividad Investigadora) y ANEP (Agencia Nacional de Evaluación y Prospectiva).

\section{Discusión y conclusiones}

La necesidad de encontrar criterios unánimemente aceptados por la comunidad científica internacional ha derivado en la adopción de los índices y bases bibliográficas y bibliométricas internacionales como los únicos indicadores de calidad para el docente universitario que someta su investigación a estos tres organismos. Los índices nacionales, como el ya desaparecido INRECS (Índice de impacto de las Revistas Españolas de Ciencias Sociales) entre otros, ocupan un lugar secundario en la evaluación.

Se confirma, por tanto, que las bases de datos internacionales son la piedra angular de la evaluación científica en el ámbito español, “[...] puesto que para la mayoría de evaluadores sólo parece existir su indicador estrella, el Impact Factor, que es publicado anualmente en los Journal Citation Reports (JCR)" (Aguillo, 2014, p. 116). La JCRdependencia, como han denominado Ruíz-Pérez, Martín-Martín y Delgado-LópezCózar (2015), es una constante en la evaluación de la actividad científica en España.

Esta situación contrasta con la realidad del área del Ciencias Sociales, en la que la mayoría de los autores publican principalmente en castellano (aunque cada vez existen más revistas internacionales que aceptan textos en castellano y revistas españolas que están indexadas en SCOPUS o Journal Citation Reports) y fundamentalmente en medios 
nacionales, bien sean revistas, actas de congresos o editoriales (Delgado-López-Cózar et al., 2014). Sin embargo, el sector editorial español de revistas en Ciencias Sociales presenta grandes debilidades, como por ejemplo, el gran número de publicaciones que cuentan con únicamente uno o dos números al año, con escaso impacto y de carácter institucional (Rodríguez-Yunta y Giménez-Toledo, 2013).

Asimismo, tal como ha señalado Giménez-Toledo (2014), "en el campo de las Humanidades y las Ciencias Sociales, son innumerables los resultados científicos que apuntan las limitaciones del indicador para evaluar la producción científica y, en consecuencia, lo inconveniente que resulta su utilización. En ninguno de los casos, esas evidencias no han servido para que se establezcan otros indicadores distintos" (p. 20). Por tanto, se duda de la idoneidad de este criterio para el área de las Ciencias Sociales en el ámbito español.

Las tres entidades adoptan el índice de impacto (factor de impacto en JCR y Scimago Journal Rank en SCOPUS) de la revista científica como el principal indicador de calidad, independientemente de juzgar la calidad relativa de cada uno de los artículos individuales publicados en ella. Por una parte habría que valorar el impacto de la publicación científica y por otra el del artículo publicado en la misma, desvinculando el factor de impacto de la publicación y el del artículo.

Otro aspecto a mejorar en futuros sistemas de evaluación, relacionado con el anterior, sería una aplicación más adecuada del análisis de las citas de los trabajos publicados, un indicador de calidad adoptado por los tres organismos. Al margen de cuestiones derivadas del significado de este indicador (Zych y Buela-Casal, 2012) o de las críticas sobre el cálculo del factor de impacto (por ejemplo: las limitaciones de cobertura y exactitud de las bases de datos del antiguo ISI, la metodología empleada para el cálculo del factor impacto, y la incorrecta interpretación de este indicador entre otros) (Borrego y Urbano, 2006), tendría que valorarse también la calidad de las mismas. La cuantificación de las citas de los trabajos publicados en las publicaciones científicas no puede considerarse el único indicador válido del impacto de la publicación individual ya que puede estar claramente manipulado, de ahí que debería estar complementado con otros criterios objetivos.

Respecto a la valoración de los libros como aportaciones resulta llamativo que en un área como las Ciencias Sociales, en la que de forma habitual se difunden los resultados de las investigaciones a través de este medio, queden relegados e incluso lleguen a no tenerse en cuenta para la evaluación, tal como ocurrió en la última convocatoria de la CNEAI en el campo de Ciencias Económicas y Empresariales. Respecto a los indicadores de calidad de los libros sería interesante, además de los ya propuestos, incluir también listados de editoriales en función de su calidad, como por ejemplo SPI (Scholarly Publishers Indicators) incorporado en la última convocatoria de la CNEAI en los campos 10 (Historia, Geografía y Artes) y 11 (Filosofía, Filología y Lingüística) $\mathrm{u}$ otros listados similares.

Se plantean también otros interrogantes como si se incorporará como criterio de calidad la inclusión de las revistas en la base de datos de Scielo Citation Index integrada en Web of Science desde Febrero de 2014, la valoración del Google Scholar Metrics y el índice h5 o si SCOPUS (con su indicador Scimago Journal Rank) ganará peso en las posteriores convocatorias de evaluación de otros campos dentro de las Ciencias 
Sociales. En definitiva, la medición de la cantidad y calidad de la labor investigadora, tal como sostienen Delgado y Fernández-Llera (2012), pasa por la necesidad de buscar indicios de calidad.

Además, en un horizonte cercano se plantean dos cambios que afectarán a la evaluación del profesorado universitario español, y en consecuencia a los indicadores de calidad seleccionados. En primer lugar, la inminente reforma del sistema de acreditación nacional propuesto por ANECA y anunciada para el segundo semestre de 2015 supondrá previsiblemente una nueva búsqueda y formulación de los indicadores de calidad de las publicaciones científicas (Proyecto de R.D. por el que se modifica el R.D. 1312/2007, por el que se establece la acreditación nacional para el acceso a los cuerpos docentes universitarios).

En segundo lugar, la transformación de ANECA en un organismo autónomo que asumirá las competencias encomendadas a la CNEAI centralizará las funciones de acreditación y evaluación tras la entrada en vigor de la Ley 15/2014, de 16 de septiembre de racionalización del Sector Público y otras medidas de reforma administrativa. Será interesante entonces analizar la idoneidad de los nuevos criterios para el ámbito de las ciencias sociales, identificar las carencias y errores y observar si los demás organismos de evaluación los toman como referencia y adoptan en sus propios procesos evaluadores.

\section{Referencias}

Agencia Nacional de Evaluación de la Calidad y Acreditación [ANECA] (2008). Principios y orientaciones para la aplicación de los criterios de investigación. Recuperado de http://www.aneca.es/Programas/ACADEMIA/ACADEMIA-instrucciones-para-la solicitud/Documentos-del-Programa

Agencia Nacional de Evaluación de la Calidad y Acreditación [ANECA] (2012). Principios y orientaciones 2.0. Recuperado de http://biblioteca.uam.es/economicas/ documentos/Principiosyorientaciones20.pdf

Agencia Nacional de Evaluación y Prospectiva [ANEP] (2007). Criterios de evaluación de la I+D en Ciencias Sociales. Recuperado de http://www.idi.mineco.gob.es/stfls/ MICINN/Investigacion/FICHEROS/2007-criterios-evaluacion-ccss3.pdf

Aguillo, I. F. (2014). Políticas de información y publicación científica. El profesional de la información, 23(2), 113-118. doi: http://dx.doi.org/10.3145/epi.2014.mar.02.

Borrego, A., \& Urbano, C. (2006). La evaluación de revistas científicas en Ciencias sociales y Humanidades. Información, Cultura y Sociedad, 14, 11-27.

Cancelo, M., \& Bastida, M. (2013). La evaluación de la investigación en España: los sexenios en las áreas de economía y empresa. CIRIEC-España, Revista de Economía Pública, Social y Cooperativa, 78, 265-292.

Caride, J. A. (2011). La evaluación-acreditación de la investigación en España: la experiencia de la Agencia Nacional de Evaluación y Prospectiva (ANEP). Educar, 47(1), 165-179.

Castro, J., Barrenechea, J., \& Ibarra, A. (2006). Indicadores de actividad científica en Ciencias Sociales y Humanidades: hacia un modelo de evaluación integral basado en patrones disciplinarios de investigación. En A. Ibarra, J. Castro y J. Barrenechea 
(Eds.), La evaluación de la actividad científica en Ciencias Sociales y Humanidades (pp. 73-103). Bilbao: Servicio Editorial de la UPV/EHU.

Delgado-López-Cózar, E., Orduña-Malea, E., Jiménez-Contreras, E., \& Ruiz-Pérez, R. (2014). H Index Scholar: el índice h de los profesores de las universidades públicas españolas en humanidades y ciencias sociales. El profesional de la información, 23(1), 87-94.

Delgado-López-Cózar, E., \& Ruiz-Pérez, R. (2009). La comunicación y edición científica fundamentos conceptuales. En C. García Caro y J. Vílchez Pardo (Coord.) Homenaje a Isabel de Torres Ramírez: Estudios de documentación dedicados a su memoria (pp. 131150). Granada: Editorial Universidad de Granada.

Delgado F. J., \& Fernández-Llera, R. (2012). Sobre la evaluación del profesorado universitario (especial referencia a ciencias económicas y jurídicas). Revista Española de Documentación Científica, 35(2), 361-375.

Galán, A., González-Galán, M.A., \& Rodríguez-Patrón, P. (2014). La evaluación del profesorado universitario en España: sistema nacional y divergencias territoriales. Revista de Educación, 366, 136-164.

Galán, A., \& Zych, I. (2011). Análisis de los criterios de la Comisión Nacional Evaluadora de la Actividad Investigadora (CNEAI) para la concesión de los tramos de investigación en Educación. Bordón. Revista de pedagogía, 63(2), 117-140.

Giménez-Toledo, E. (2014). Imposturas en el ecosistema de la publicación científica. Revista de Investigación Educativa, 32(1), 13-23. doi: http://dx.doi.org/10.6018/ rie.32.1.19025

Hernández, F., \& Maquilón, J.J. (2010). Indicadores de calidad de las revistas científicas y sistema de gestión editorial mediante OJS. Revista de Investigación Educativa, 28(1), 13-29.

Jiménez-Contreras, E., De Moya Anegón, F., \& Delgado-López-Cózar, E. (2003). The evolution of research activity in Spain. The impact of National Commisssion for the Evaluation of Research Activity (CNEAI). Research Policy, 32(1), 123-142.

Ley 15/2014, de 16 de septiembre de racionalización del Sector Público y otras medidas de reforma administrativa.

Masip, P. (2011). Efecto Aneca: producción española en comunicación en el Social Science Sitation Index. Anuario ThinkEPI, 5, 206-210.

Moreno-Pulido, A., López-González, M. Á., Rubio-Garay, F., Ángel Saúl, L., \& SánchezElvira-Paniagua, Á. (2013). Evolución de las revistas españolas de Ciencias Sociales en el Journal Citation Reports (2006-2010) y su valoración como indicio de calidad en la normativa evaluadora española. Revista Española de Documentación Científica, 36(3). doi: http://dx.doi.org/10.3989/redc.2013.3.987

Ortiz-de-Urbina-Criado, M., \& Mora-Valentín, E-M. (2013). El sistema de acreditación del profesorado a través del Programa ACADEMIA: Evolución y cambios. Revista Española de Documentación Científica, 36(1). doi: http://dx.doi.org/10.3989/redc.2013.1.971

Perceval, J. M., \& Fornieles, J. (2008). Confucio contra Sócrates: la perversa relación entre la investigación y la acreditación. Anàlisi, 36, 213-224.

Proyecto de R.D. por el que se modifica el R.D. 1312/2007, por el que se establece la acreditación nacional para el acceso a los cuerpos docentes universitarios. 
Resolución de 26 de noviembre de 2014, de la Comisión Nacional Evaluadora de la Actividad Investigadora, por la que se publican los criterios específicos aprobados para cada uno de los campos de evaluación. Boletín Oficial del Estado de 1 de diciembre de 2014, 290, 98204-98219. Disponible en: http://www.boe.es/diario_boe/ txt.php?id=BOE-A-2014-12482

Rodríguez-Yunta, L., \& Giménez-Toledo, E. (2013). Fusión, coedición o reestructuración de revistas científicas en humanidades y ciencias sociales. El profesional de la información, 22(1), 36-45. doi: http://dx.doi.org/10.3145/epi.2013.ene.05

Rovira, L. (2006). ¿Hacia una evaluación métrica de la investigación en las humanidades y en las ciencias sociales?. En A. Ibarra, J. Castro, J. Barrenechea (Eds.), La evaluación de la actividad científica en Ciencias Sociales y Humanidades (pp. 31-52). Bilbao: Servicio Editorial de la UPV/EHU.

Ruíz-Pérez, R., Delgado-López-Cózar, E., \& Jiménez-Contreras, E. (2010). Principios y criterios utilizados en España por la Comisión Nacional Evaluadora de la Actividad Investigadora (CNEAI) para la valoración de las publicaciones científicas: 1989-2009. Psicothema, 22(4), 898-908.

Ruíz-Pérez, R., Martín-Martín, M., \& Delgado-López-Cózar, E. (2015). Las revistas universitarias en el marco de los criterios de evaluación de la actividad investigadora en España. Revista Española de Documentación Científica, 38(2). doi: http://dx.doi. org/10.3989/redc.2015.2.1191

Soriano, J. (2008). El efecto ANECA. En Actas y memoria final. Congreso internacional fundacional AE-IC (pp.1-18). Santiago de Compostela: Asociación Española de Investigación de la Comunicación.

Torres-Salinas, D., Bordons, M., Giménez-Toledo, E., Delgado-López-Cózar, E., JiménezContreras, E., \& Sanz-Casado, E. (2010). “Clasificación integrada de revistas científicas $(C I R C)$ : propuesta de categorización de las revistas de ciencias sociales y humanas". El profesional de la información, 19(6), 675-683. doi: 10.3145/epi.2010.nov.1510.3145/ epi.2014.mar.02

Zych, I., \& Buela-Casal, G. (2012). What do the scientists think about the impact factor? Scientometrics, 92(2), 281-292.

Fecha de recepción: 13/10/2014

Fecha de revisión: 15/10/2014

Fecha de aceptación: 27/09/2015 